\title{
Psicologia Social, Organizacional e do Trabalho Estrutura Fatorial e Propriedades Psicométricas da Escala de Suporte à Transferência de Treinamento
}

\author{
Lara Barros Martins* \\ Universidade de São Paulo, São Paulo, SP, Brasil \\ Universidad de Sevilla, Sevilla, Espanha \\ Thaís Zerbini \\ Universidade de São Paulo, São Paulo, SP, Brasil \\ Francisco J. Medina \\ Universidad de Sevilla, Sevilla, Espanha
}

\begin{abstract}
RESUMO - O estudo objetivou analisar a estrutura fatorial e as propriedades psicométricas da Escala de Suporte à Transferência de Treinamento (ESTT) e sua relação com a efetividade do treinamento no trabalho. Um total de 1.540 funcionários de um banco estatal brasileiro participou do estudo respondendo à ESTT e à escala de impacto do treinamento no trabalho em amplitude depois de realizar um curso online. Foram realizadas análises de consistência interna, fatorial confirmatória e de regressões múltiplas. Uma estrutura com três fatores foi encontrada, bem como um alto nível de ajuste para os modelos. As medidas apresentam evidências de validade de construto e o suporte percebido pelos participantes esteve positivamente relacionado com a transferência de competências para o trabalho e desempenho geral.
\end{abstract}

Palavras-chave: educação à distância, avaliação, suporte à transferência, escala, efetividade de treinamento

\section{Factor Structure and Psychometric Properties of the Scale of Support for Training Transfer}

\begin{abstract}
This study aimed to analyze the factor structure and psychometric properties of a Training Transfer Support Scale (STTS) and its relationship with the effectiveness of training at work. A total of 1,540 employees of a public Brazilian bank participated in this study by responding to the STTS and a measure of transfer of training after taking part in an online course. Reliability analysis, confirmatory factor analysis, and multiple regressions were conducted. A three-factor structure was found and a high level of fit for the models. Evidence of the construct validity of the measures was obtained, and the participants' perception of support was positive related to the transfer of training to the workplace and overall performance.
\end{abstract}

Keywords: e-learning, assessment, support to training transfer, scale, effectiveness of training

O desenvolvimento de medidas válidas e confiáveis contribui para que sejam realizados avanços no campo teórico-científico, produzindo conhecimentos sobre os fatores que operam para afetar a aprendizagem e a performance no trabalho (Aguinis \& Kraiger, 2009). Tal desenvolvimento também traz implicações práticas no que se refere às intervenções feitas no contexto corporativo.

Características organizacionais e do ambiente social no trabalho influenciam os resultados de treinamento e podem interferir no desempenho (Abbad, Zerbini, \& Souza, 2010; Alvarez, Salas, \& Garofano, 2004; Meneses, Zerbini, \& Martins, 2012; Salas \& Cannon-Bowers, 2001; Tannenbaum \& Yukl, 1992). Entre as variáveis contextuais que apresentam os mais fortes e consistentes relacionamentos com o impacto do treinamento no trabalho, destaca-se o Suporte à Transferência, que avalia o apoio recebido por egressos de

* Endereço para correspondência: Faculdade de Filosofia, Ciência e Letras de Ribeirão Preto, Universidade de São Paulo, Departamento de Psicologia, Av. Bandeirantes, 3900, Bairro Monte Alegre, Ribeirão Preto, SP, Brasil. CEP: 14.040-901. E-mail: lara_bmartins@hotmail.com treinamentos para aplicar as novas habilidades adquiridas no trabalho (Abbad et al., 2012).

O construto compreende características situacionais importantes, sendo o apoio material e psicossocial, os principais fatores estudados em pesquisas. O suporte psicossocial refere-se ao apoio gerencial e social (colegas ou pares) à aplicação de novas aprendizagens no trabalho, que se dá por meio do estabelecimento de metas, oferecimento de assistência (discutir e encorajar o uso de novas competências no trabalho) e feedback (Abbad et al., 2012; Burke \& Hutchins, 2007). O suporte material, por sua vez, avalia os elementos de apoio ambiental oferecidos pela organização, mensurando a qualidade, a suficiência e a disponibilidade de recursos materiais e financeiros disponíveis e a adequação do ambiente físico do local de trabalho à aplicação no trabalho das novas competências adquiridas em treinamento (Abbad et al., 2012).

Desde que Baldwin e Ford (1988) identificaram o suporte como um componente do ambiente de trabalho fundamental para a manutenção de competências e o processo de transferência de treinamento, pesquisas realizadas em contextos, treinamentos e amostras de participantes 
diferentes vêm confirmando o papel importante exercido pelo suporte à transferência na explicação de impacto de treinamento no trabalho. A existência de correlações fortes e moderadas entre essas variáveis mostra que a aplicação de novas aprendizagens no trabalho depende do apoio oferecido pela organização e pelos seus membros (chefes e pares) aos egressos de treinamentos (Abbad et al., 2012; Abbad, Pilati, \& Pantoja, 2003; Aguinis \& Kraiger, 2009; Burke \& Hutchins, 2007; Joo, Lim, \& Park, 2011).

Ambos os tipos de suporte, gerencial e de pares, influenciam significativamente a propensão dos treinandos para utilizar as novas competências no local de trabalho (Al-Eisa, Furayyan, \& Alhemoud, 2009; Dermol \& Čater, 2013; Grossman \& Salas, 2011; Nijman, Nijhof, Wognum, \& Veldkamp, 2006; Pham, Segers, \& Gijselaers, 2012), sendo os preditores mais consistentes de resultados de impacto do treinamento no trabalho encontrados (Burke \& Hutchins, 2007; Chiaburu, van Dam, \& Hutchins, 2010). Por um lado, os estudos enfatizam a importância do envolvimento e da participação dos supervisores no treinamento para propiciar resultados de transferência de treinamento (Blume, Ford, Baldwin, \& Huang, 2010; Grossman \& Salas, 2011), pois a sua postura e opinião afetam os treinandos (Salas, Tannenbaum, Kraiger, \& Smith-Jentsch, 2012). Há recomendações de que se deve considerar a possibilidade de avaliar não somente as dimensões de supervisão póstreinamento (por exemplo, reuniões e feedback), mas também as intervenções dos supervisores antes ou durante o treinamento que podem ter influência sobre o impacto do treinamento no trabalho (Velada, Caetano, Michel, Lyons, \& Kavanagh, 2007).

Antes do início do treinamento, os supervisores devem guiar e preparar os treinandos para o treinamento certo e esclarecer as suas expectativas. Para tanto, os supervisores devem ser envolvidos no processo prévio de avaliação de necessidades para que entendam a necessidade do treinamento e possam fornecer informações precisas e motivadoras sobre o mesmo (Salas et al., 2012), comunicar os objetivos com relação ao desempenho desejado, as condições sob as quais o desempenho deverá ocorrer no local de trabalho e os critérios de desempenho considerado aceitável (Burke \& Hutchins, 2007). Após o término do treinamento, os supervisores devem adotar uma postura positiva frente ao mesmo, remover obstáculos e garantir ampla oportunidade para que os egressos possam aplicar o que aprenderam e receber feedback (Salas et al., 2012).

Por outro lado, em algumas pesquisas, o apoio de pares mostrou-se mais forte preditor do impacto do treinamento no trabalho do que os demais (gerencial e organizacional). Isso se deve talvez pelo contato direto estabelecido, mesmo status funcional e contínuo fluxo de informações entre colegas de trabalho (Chiaburu, 2010). Contudo, ainda há poucas evidências sugerindo que um tipo de apoio exerce uma influência maior sobre o impacto do treinamento no trabalho do que o outro, ou seja, supervisor versus pares (Grossman \& Salas, 2011; Van den Bossche, Segers, \& Jansen, 2010).

As investigações mostram o papel importante desses fatores interpessoais enquanto moderadores da relação entre treinamento-impacto do treinamento no trabalho (Aguinis \& Kraiger, 2009; Burke \& Hutchins, 2007). Esses suportes mais próximos e concretos, em comparação ao mais distante e difuso, provindo da organização, possuem influências positivas sobre o impacto do treinamento no trabalho, ao lado de outros fatores individuais (motivação, autoeficácia, orientação de metas para a aprendizagem etc.) (Chiaburu et al., 2010). Esses achados indicam a grande importância das relações humanas ante as condições materiais para o sucesso do processo de transposição do aprendido em treinamentos para o ambiente de trabalho.

Ao passo que os suportes à transferência recebidos por egressos de treinamentos para aplicar as novas habilidades adquiridas no trabalho são comprovadamente fatores que mantêm relacionamento com o impacto do treinamento no trabalho, sendo preditores que influenciam na sua explicação, nós elaboramos um instrumento capaz de mensurá-los, tendo como base principalmente a escala de Suporte à Transferência de Treinamento de Abbad e Sallorenzo (2001). Outras escalas também foram consultadas como exemplos para a criação dessa nova versão, como a de Suporte Gerencial de Yarnall (1998; estrutura unifatorial com 5 itens, $\alpha=$ $0,91)$, posteriormente revalidada por Chiaburu e Tekleab (2005) (7 itens, $\alpha=0,95$ ); e a de Tracey e Tews (2005), General Training Climate Scale (GTCS; 15 itens, 3 fatores de suporte: gerencial, trabalho e organizacional, $0,85<\alpha<$ $0,87)$. Neste caso, apesar do construto avaliado ser clima para transferência, esta variável é correlata a suporte (Abbad et al., 2012), e o instrumento faz alusão e pretende medir aspectos relativos ao que se denomina suporte, como é observado pela própria nomeação dos fatores obtidos, contendo itens bastante similares aos das escalas anteriormente citadas.

O objetivo deste estudo foi analisar a estrutura fatorial e as características psicométricas da Escala de Suporte à Transferência de Treinamento (ESTT) em um contexto organizacional a distância. Justifica-se a confirmação da estrutura fatorial da ESTT pois várias pesquisas nacionais vêm utilizando a medida (e.g., Carvalho \& Abbad, 2006; Pilati \& Borges-Andrade, 2012), a qual manteve, continuamente, boas qualidades psicométricas e excelentes índices de consistência interna. Contudo, todos os estudos possuíam caráter exploratório e, portanto, não realizaram a análise fatorial confirmatória, mantendo esse passo como agenda de pesquisa. Objetivou-se, também, atestar a capacidade concorrente da medida para que esta seja utilizada em outros contextos para a avaliação do suporte à transferência de treinamento.

\section{Método}

\section{Participantes}

Participaram do estudo funcionários de um banco estatal brasileiro, que haviam realizado durante a jornada de trabalho um treinamento online e autoinstrucional sobre o tema da promoção de eficiência operacional nas atividades de trabalho, denominado "Eficiência Operacional". As respostas obtidas quanto à caracterização demográfica e funcional, de 1.639 respondentes, indicam que a maioria: é do sexo masculino (56,8\%), entre 46 e 55 anos de idade 
(26,1\%), trabalha na Unidade de Apoio aos Negócios e à Gestão (37,8\%), possui experiência na função de 1 a 3 anos $(20,3 \%)$, e com Graduação $(33,6 \%)$.

Instrumentos

O instrumento ESTT contém 14 itens, associados a uma escala de frequência que varia de 1 ("Nunca") a 5 ("Sempre"), que mensuram o suporte oferecido por supervisores (6 itens), pares (6 itens) e o suporte material fornecido pela organização ( 2 itens) à aplicação no trabalho das novas competências aprendidas em treinamento. Os seis itens referentes ao apoio de supervisores e de pares são idênticos na redação, mas devem ser respondidos em suas colunas correspondentes (supervisores e pares) no questionário (ver Apêndice). Foi adaptado da versão proposta por Abbad e Sallorenzo (2001), composta por duas dimensões: Suporte Psicossocial à Transferência (11 itens, $\alpha=0,91$; cargas fatoriais: 0,46 a 0,86$)$ e Suporte Material à Transferência $(5$ itens, $\alpha=0,86$; cargas fatoriais: 0,56 a 0,82 ). A escala passou pela avaliação de pesquisadores doutores especialistas da área $(n=2)$, em processo de validação por juízes, para identificar e, eventualmente, corrigir possíveis falhas presentes no instrumento de medida e julgar sua adequação teórica.

Para medir o impacto do treinamento no trabalho em amplitude foi utilizada uma versão adaptada da Escala de Impacto no Treinamento no Trabalho (Pilati \& Abbad, 2005), com 7 itens $(\alpha=0,89$ e cargas fatoriais entre 0,62 e 0,86$)$ e alternativas de resposta pontuadas de 1 ("Não concordo") a 5 ("Concordo totalmente"). O impacto em amplitude referese à influência indireta do treinamento sobre o desempenho global, atitudes e motivação dos egressos (exemplos de itens: "Realizo meus trabalhos com mais agilidade", "Melhorou a qualidade do meu trabalho", "Aumentou minha motivação para o trabalho").

\section{Procedimento de Coleta e de Análise de Dados}

A coleta de dados foi realizada totalmente a distância, por meio da disponibilização conjunta e virtual dos questionários na página da Universidade Corporativa do Banco a uma amostra potencial de 3.600 funcionários. Tais funcionários haviam participado do curso mencionado, após seis meses a um ano a partir do final do treinamento, para possibilitar que seus efeitos pudessem ser observados no nível individual, em termos de desempenhos gerais no trabalho.

Para executar as análises, o SPSS (Statistical Package for the Social Science) e SPSS 22.0 AMOS foram utilizados. Análises preliminares foram realizadas para a identificação de dados ausentes (presentes em menos de 5\% dos casos) e extremos univariados (inexistência de outliers), e se respeitavam o pressuposto da normalidade (assimetria e curtose entre $-2,0$ e 2,0 ).

Os 1.540 casos válidos de respostas a ESTT foram submetidos a análises fatoriais confirmatórias (AFC; Método de Máxima Verossimilhança) e de consistência interna (Alfa de Cronbach: $\alpha$ ). Para julgar a bondade de ajuste do modelo, as medidas incrementais de ajuste (Goodness-ofFit Index [GFI], Comparative-Fit Index [CFI] e Tucker Luwis Index [TLI]) deveriam ter valores superiores a 0,90 (idealmente $>0,95$ ) e as residuais (Root Mean Square
Residual [RMSR] e Root Mean Square Error Approximation [RMSEA]), inferiores a 0,08 (idealmente $<0,05$ ). $\mathrm{O}$ valor do qui-quadrado sobre graus de liberdade $\left(\chi^{2} / g l\right.$ ou CMIN/ $D F$ ) deveria ser inferior a 5 - contudo, amostras muito grandes interferem sobre esse valor, que deixa de ser um bom indicador de ajuste do modelo (Rios \& Wells, 2014). Nos casos em que se obteve valores superiores ao aceitável, os outros índices citados foram levados em consideração. O índice de parcimônia Bayesian Information Criterion (BIC) foi usado para comparar os ajustes entre modelos (Byrne, 2010). Para analisar a validade concorrente da escala, utilizou-se a análise de regressão múltipla, com os três fatores do ESTT como preditores da efetividade do treinamento no trabalho (impacto em amplitude).

\section{Resultados}

\section{Análise Teórica}

As sugestões provenientes da validação por juízes consistiram em pequenas alterações no layout do instrumento e na redação do item 8 ("O local de trabalho é adequado à aplicação eficaz das novas habilidades aprendidas em treinamento"), que foi modificada para "É disponibilizado local adequado à aplicação eficaz das novas habilidades aprendidas em treinamento", mantendo o paralelismo entre os demais itens, que começam com verbos na terceira pessoa do plural para referir-se aos supervisores e pares.

\section{Análises Descritivas}

Como pode ser observado na Tabela 1, a frequência com que supervisores, pares e organização ofereceram os apoios descritos nos itens, segundo a percepção dos funcionários treinados, não variou muito entre si, sendo as médias bastante próximas (escala de 5 pontos): de 3,62 $(D P=1,12)$ a 3,94 (DP $=1,01)$ (supervisores); 3,42 $(D P=1,08)$ a 3,70 $(D P=1,02)$ (pares); e 3,70 $(D P=1,00)$ a $3,77(D P=1,00)$ (material). Por esse motivo, os escores médios foram comparados por meio de Paired-Sample T Test, tendo sido obtidos os seguintes resultados para cada par: 1) Suporte Supervisores-Suporte Pares: $t=9,07 ; d f=1291 ; p<0,0001(95 \% C I=[+0,13$; $+0,21])$; 2) Suporte Supervisores-Suporte Material: $t=2,69$; $d f=1259 ; p<0,007(95 \% \mathrm{CI}=[+0,01 ;+0,11] ; 3)$ Suporte Pares-Suporte Material: $t=-3,75 ; d f=1212 ; p<0,0001(95 \%$ $\mathrm{CI}=[-0,15 ;-0,04])$.

Nota-se, por exemplo, que o apoio de supervisores e pares no incentivo ao uso no trabalho das novas competências desenvolvidas em treinamento (item 1) foi percebido de maneira similar pelos respondentes, sendo bastante frequente. Por outro lado, o fornecimento de feedback para a aplicação no trabalho das novas habilidades adquiridas em treinamento (item 5) apresentou a menor média, tendo sido o apoio menos frequentemente percebido pelos funcionários, tanto advindo de seus supervisores, quanto de seus pares. 
Tabela 1. Estatísticas descritivas do instrumento ESTT

\begin{tabular}{|c|c|c|c|}
\hline $\begin{array}{l}\text { Tipo de } \\
\text { Suporte }\end{array}$ & Itens & $X$ & $D P$ \\
\hline \multirow{6}{*}{ 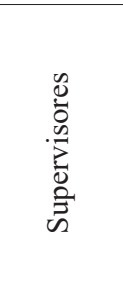 } & 1. Encorajam a aplicação no trabalho de novas habilidades aprendidas em treinamento. & 3,94 & 1,01 \\
\hline & 2. Fornecem as informações necessárias ao uso eficaz das novas habilidades aprendidas em treinamento. & 3,86 & 1,00 \\
\hline & 3. Removem as dificuldades ao uso eficaz das novas habilidades aprendidas em treinamento. & 3,79 & 0,99 \\
\hline & 4. Criam oportunidades de usar no trabalho as novas habilidades aprendidas em treinamento. & 3,74 & 1,02 \\
\hline & 5. Fornecem feedback quanto à aplicação no trabalho de novas habilidades adquiridas em treinamento. & 3,62 & 1,12 \\
\hline & 6. Consideram minhas sugestões, em relação ao que foi aprendido em treinamento, no trabalho. & 3,74 & 1,03 \\
\hline \multirow{6}{*}{ 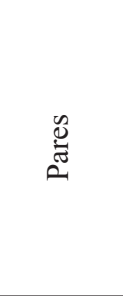 } & 1. Encorajam a aplicação no trabalho de novas habilidades aprendidas em treinamento. & 3,70 & 1,02 \\
\hline & 2. Fornecem as informações necessárias ao uso eficaz das novas habilidades aprendidas em treinamento. & 3,66 & 0,99 \\
\hline & 3. Removem as dificuldades ao uso eficaz das novas habilidades aprendidas em treinamento. & 3,63 & 0,96 \\
\hline & 4. Criam oportunidades de usar no trabalho as novas habilidades aprendidas em treinamento. & 3,58 & 1,00 \\
\hline & 5. Fornecem feedback quanto à aplicação no trabalho de novas habilidades adquiridas em treinamento. & 3,42 & 1,08 \\
\hline & 6. Consideram minhas sugestões, em relação ao que foi aprendido em treinamento, no trabalho. & 3,68 & 0,98 \\
\hline \multirow{2}{*}{ 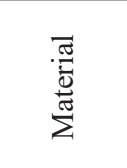 } & $\begin{array}{l}\text { 7. São fornecidos os recursos materiais necessários para a aplicação eficaz das novas habilidades } \\
\text { aprendidas em treinamento. }\end{array}$ & 3,77 & 0,89 \\
\hline & $\begin{array}{l}\text { 8. É disponibilizado local de trabalho adequado à aplicação eficaz das novas habilidades aprendidas em } \\
\text { treinamento (por exemplo, espaço físico, iluminação, mobiliário, nível de ruído). }\end{array}$ & 3,70 & 1,00 \\
\hline
\end{tabular}

As correlações entre os três fatores da ESTT e os valores do d de Cohen (tamanho do efeito) podem ser visualizados na Tabela 2 .

\section{Análise Fatorial Confirmatória e Consistência Interna}

Como todos os itens apresentam distribuição normal, tendo seus valores de assimetria e curtose aceitáveis (assimetria de

Tabela 2. Correlações entre os fatores do instrumento ESTT

\begin{tabular}{lccc}
\hline \multicolumn{1}{c}{ Variável } & SS & SP & SM \\
\hline Suporte Supervisores [SS] & - & $\mathrm{d}=0,19$ & $\mathrm{~d}=0,06$ \\
Suporte Pares [SP] & $0,702 *$ & - & $\mathrm{d}=0,12$ \\
Suporte Material [SM] & $0,481^{*}$ & $0,424^{*}$ & - \\
Média & 3,81 & 3,64 & 3,75 \\
Desvio-padrão & 0,90 & 0,87 & 0,87 \\
\hline
\end{tabular}

Nota. Todas as correlações são significativas * $p<0,01 ; d=\mathrm{d}$ de Cohen

$-0,92$ a -0,41, e curtose de -0,26 a 0,58), procedeu-se à AFC, usando o Método de Máxima Verossimilhança. O modelo hipotético inicial (estrutura empírica com 3 fatores, 14 itens e $0,85<\alpha<0,95)$ apresenta falhas na bondade de ajuste (Tabela 3 e Figura 1), indicando a necessidade de observar os índices de modificação e introduzir as re-especificações.

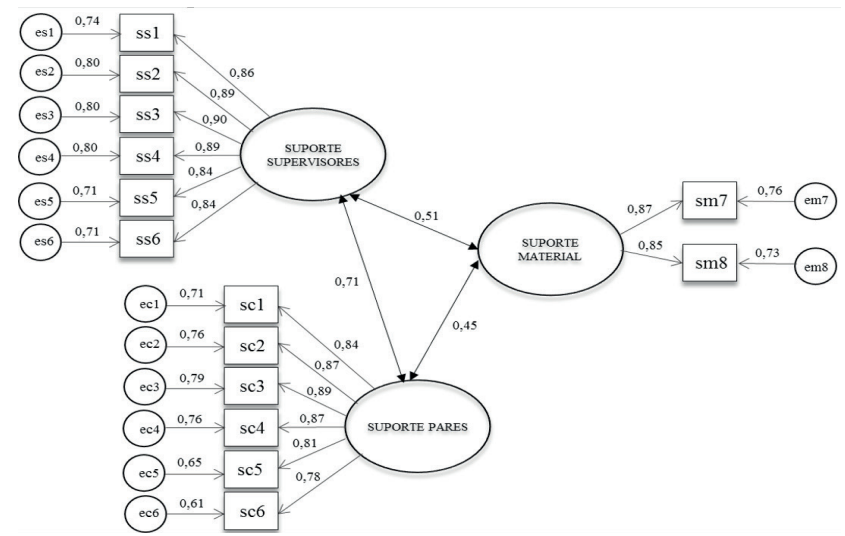

Figura 1. Cargas fatoriais estandardizadas, coeficientes de correlação e erros padrão da AFC para o instrumento de Suporte à Transferência de Treinamento (modelo hipotético inicial).

Logo, com a indicação da presença de multicolinearidade entre os mesmos itens presentes nos fatores de suporte de supervisores e de pares ("Removem as dificuldades ao uso eficaz das novas habilidades aprendidas em treinamento" e "Criam oportunidades de usar no trabalho as novas habilidades aprendidas em treinamento") - itens 3 e 4 (supervisores: $r=$ 0,82 ) e itens 9 e 10 (pares: $r=0,80$ ) - optou-se por testar o modelo com a retirada dos itens 3 e 9 , já que há altos indícios de que os participantes consideraram similares os atos de "remover dificuldades" e "criar oportunidades". Faz sentido

Tabela 3. Indicadores de ajuste para os modelos original e re-especificados do instrumento ESTT

\begin{tabular}{lcccccccc}
\hline Modelo & $\chi^{2}$ & $\boldsymbol{g l}$ & $\boldsymbol{C M I N / D F}$ & $\boldsymbol{G F I}$ & $\boldsymbol{R M S R}$ & $\boldsymbol{C F I}$ & $\boldsymbol{T L I}$ & $\boldsymbol{R M S E A}$ \\
\hline Original & 2293,272 & 74 & 30,99 & 0,82 & 0,03 & 0,89 & 0,86 & 0,14 \\
Re-especificado 1 & 1811,171 & 51 & 35,51 & 0,84 & 0,03 & 0,89 & 0,85 & 0,15 \\
Re-especificado 2 & 1708,399 & 49 & 34,86 & 0,85 & 0,03 & 0,89 & 0,86 & 0,14 \\
\hline
\end{tabular}

Notas. N=1.540; o modelo re-especificado 1 não inclui os itens 3 e 9; o modelo re-especificado 2 contém a correlação entre o par de erros e5-e6, além da exclusão dos itens 3 e 9. 
teórico supor que ao criar oportunidades para a aplicação das habilidades aprendidas é necessário que antes tenham sido removidas as dificuldades que poderiam obstaculizar o uso eficaz das novas competências no trabalho. Esse modelo (modelo re-especificado 1) apresentou valores ainda não satisfatórios para os indicadores de ajuste. Em comparação com o anterior, o índice de parcimônia BIC aponta que este, com a exclusão dos itens altamente correlacionados entre si, é mais ajustado que aquele, com os 14 itens iniciais na estrutura proposta $(\triangle B I C=511,45)$.

Com vistas a melhorar o ajuste do modelo, foram analisados os índices de modificação, que apresentaram valores importantes entre os erros e5-e6 (5 - "Fornecem feedback quanto à aplicação no trabalho de novas habilidades adquiridas em treinamento" e 6 - "Consideram minhas sugestões, em relação ao que foi aprendido em treinamento, no trabalho"), tanto para o fator referente ao apoio de supervisores quanto o de pares, indicando que os participantes provavelmente entenderam tais itens como uma mesma medida. Uma vez introduzidas as correlações entre esses pares de erros, os valores para os indicadores obtidos não foram satisfatórios (modelo re-especificado 2). Em comparação com o anterior, o índice de parcimônia BIC aponta que este, com a exclusão dos itens altamente correlacionados entre si e a inclusão das correlações entre os erros (e5-e6) em ambos fatores (supervisores e pares), é mais ajustado $(\triangle B I C=88,09)$, mas ainda não apresenta bons indicadores.

Devido à forte e positiva correlação entre os fatores de suporte de supervisores e de pares $(r=0,71)$ - talvez os fatores não deveriam ser estimados em separado, mas como uma única medida, o suporte psicossocial, como preconiza a literatura dominante da área - testou-se um modelo com somente dois fatores, suporte psicossocial (12 itens relativos ao apoio de supervisores e de pares) e suporte material (2 itens). Este modelo mostrou o pior ajuste em comparação aos anteriormente citados: $n=1.540, \chi^{2}=5769,016, g l=$ 76, $C M I N / D F=75,90, G F I=0,55, R M S E A=0,22, R M R=$ 0,09; $C F I=0,72, T L I=0,67, B I C=5981,863$ (acréscimo de 3461,06 em comparação ao modelo hipotético inicial). Ainda foram testados um modelo bifator e um de segunda ordem, mas ambos apresentaram falhas na bondade de ajuste.

Finalmente, ao testar separadamente a escala que avalia o apoio de supervisores (Figura 2) da escala de suporte de pares (Figura 3), compostas pelos seis itens iniciais - cada uma associada ao fator que mede o suporte material (2 itens) - sem a inclusão de correlações entre pares de erros ou a supressão de itens, obtiveram-se os melhores índices, bem

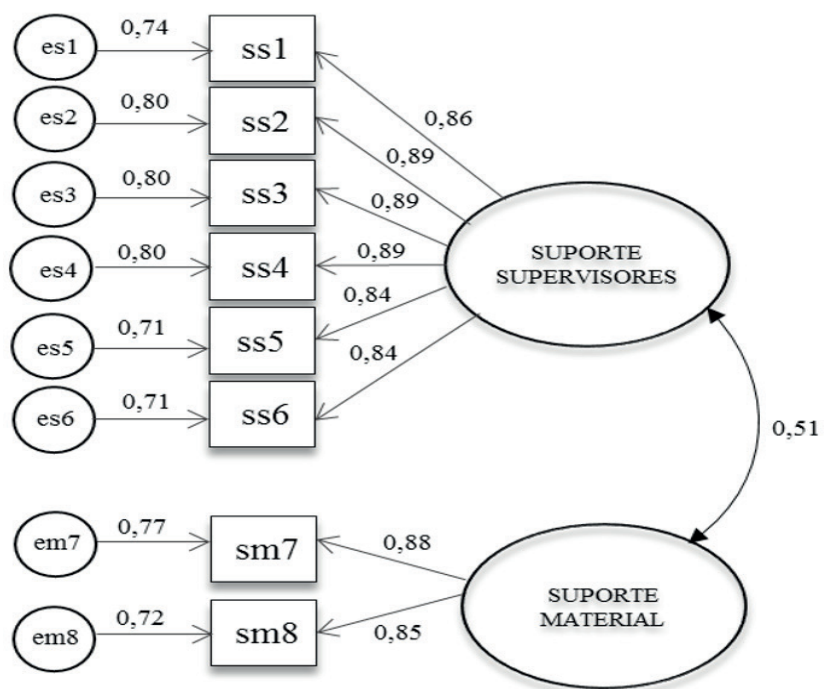

Figura 2. Cargas fatoriais estandardizadas, coeficientes de correlação e erros padrão da AFC para a escala de Suporte de Supervisores e Material do instrumento de Suporte à Transferência de Treinamento.

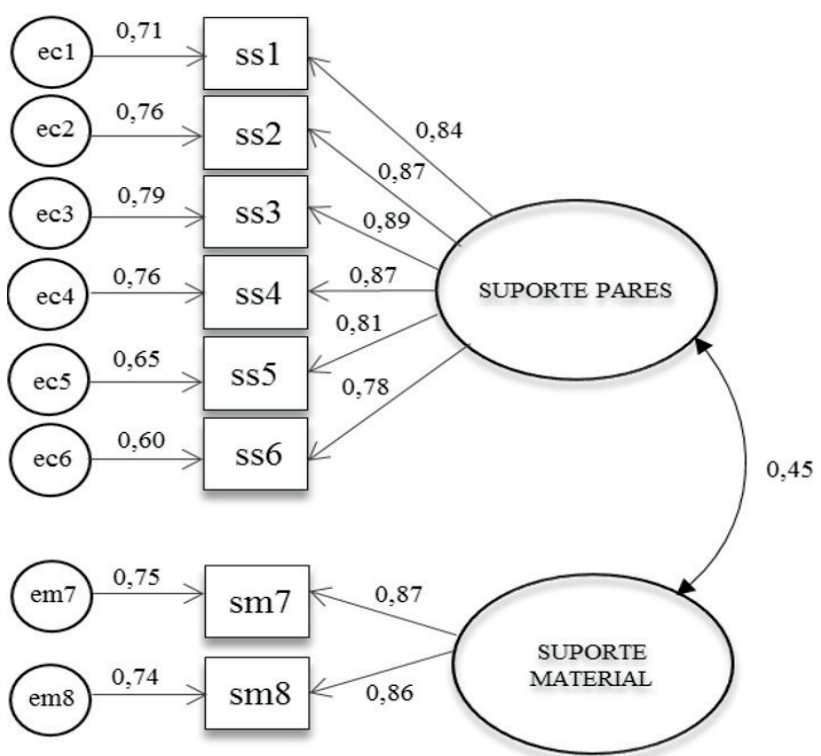

Figura 3. Cargas fatoriais estandardizadas, coeficientes de correlação e erros padrão da AFC para a escala de Suporte de Pares e Material do instrumento de Suporte à Transferência de Treinamento.

Tabela 4. Indicadores de ajuste para as escalas do instrumento ESTT

\begin{tabular}{lcccccccc}
\hline Modelo & $\chi^{2}$ & $\boldsymbol{g l}$ & $\boldsymbol{C M I N / D F}$ & $\boldsymbol{G F I}$ & $\boldsymbol{R M S R}$ & $\boldsymbol{C F I}$ & $\boldsymbol{T L I}$ & $\boldsymbol{R M S E A}$ \\
\hline Suporte Supervisores & 230,888 & 19 & 12,15 & 0,96 & 0,01 & 0,98 & 0,97 & 0,08 \\
Suporte Pares & 217,514 & 19 & 11,44 & 0,96 & 0,02 & 0,97 & 0,96 & 0,08 \\
\hline
\end{tabular}

Notas. $N=1.540$; cada modelo, além do fator de suporte supervisores ou pares, contém o fator de suporte material. 
como os valores dos resíduos baixos, como mostra a Tabela 4. O cômputo do alfa de Cronbach para as escalas, com 8 itens cada, resultou em $\alpha=0,92$ para a de suporte de supervisores/ material e $\alpha=0,90$ para a de suporte de pares/material.

\section{Influência da ESTT sobre a Efetividade de Treinamento no Trabalho}

De acordo com as estatísticas apresentadas na Tabela 5, o coeficiente de regressão múltipla $(\mathrm{R})$ foi significativamente diferente de zero, $F(3,1540)=85,343,<0,0001$, com limites de confiança de $95 \%$. Na autoavaliação do treinamento, todos os tipos de apoio contribuíram significativamente para a explicação de impacto do treinamento no trabalho em amplitude $\left(R^{2}=0,17 ; p<0,01\right)$, principalmente o suporte material ( $S M$ : $\beta=0,26 ; p<0,001)$, confirmando a influência positiva do suporte à transferência para a ocorrência do o suporte psicossocial, pois permite avaliar os dois tipos de suporte e em que medida seu oferecimento é percebido pelos egressos de treinamentos, bem como tal separação possibilita a realização de análises para verificar se há um apoio mais importante que o outro e seus efeitos sobre o impacto do treinamento no trabalho. Ou ainda, quando pertinente, avaliar somente o apoio de supervisores ou o de pares.

Os efeitos do suporte à transferência bem-sucedida das novas competências aprendidas em treinamento para o trabalho foram corroborados, uma vez que os egressos que perceberam os apoios oriundos da organização (recursos materiais, condições de trabalho etc.) e dos companheiros de trabalho (supervisores e pares) tiveram seus desempenhos gerais modificados. Esses resultados confirmam achados de investigações anteriores (e.g., Burke \& Hutchins, 2007; Blume et al., 2010; Van den Bossche et al., 2010) e indicam para a pertinência de se continuar medindo os suportes material e psicossocial oferecidos para a produção de consequências

Tabela 5. Suportes à transferência como preditores da efetividade de treinamento no trabalho

\begin{tabular}{|c|c|c|c|c|c|c|c|}
\hline Variável & & & Itoavaliaç & de IA & & & $\begin{array}{c}\text { Correlação } \\
\text { com IA }\end{array}$ \\
\hline Coeficientes & $\mathrm{B}$ & Erro Padrão & Beta $(\beta)$ & $\mathrm{t}$ & Sig. & $\mathrm{Sr} 2$ & $\mathrm{r}$ \\
\hline Suporte Supervisores [SS] & 0,085 & 0,025 & $0,13 *$ & 3,400 & 0,001 & 0,007 & $0,33 *$ \\
\hline Suporte Pares [SP] & 0,078 & 0,025 & $0,11 *$ & 3,122 & 0,002 & 0,006 & $0,31 *$ \\
\hline Suporte Material [SM] & 0,173 & 0,020 & $0,26^{*}$ & 8,546 & 0,000 & 0,050 & $0,37 *$ \\
\hline R2/R2ajustado & \multicolumn{6}{|c|}{$\mathrm{R} 2 /$ R2ajustado $=0,17^{*}$} & \\
\hline
\end{tabular}

fenômeno de resultado do treinamento no desempenho dos egressos. Do mesmo modo, os diferentes suportes estiveram correlacionados com o impacto em amplitude $(0,31<r$ $<0,37$ ), sobretudo, mais uma vez, aquele oferecido pela organização [SM].

\section{Discussão}

A estrutura empírica encontrada mostra-se adequada tanto teórica quanto empiricamente e valida a adaptação feita no instrumento, na qual os tipos de suporte psicossocial (supervisores e pares) foram medidos separadamente, além do suporte material oferecido pela organização. As escalas apresentam evidências de validade e fidedignidade, contemplando itens representativos de cada fator, e servem como ferramentas úteis para mensurar os diferentes tipos de apoio percebidos por participantes de treinamento, os quais afetam na transferência de competências para o trabalho segundo os resultados obtidos.

A grande modificação realizada no instrumento de Abbad e Sallorenzo (2001) foi adequar os itens com foco na ação, já que as medidas, apesar de abordarem conjuntamente o apoio de supervisores e pares como pressupõe o conceito de suporte psicossocial à transferência, o separavam em seus itens: alguns deles avaliavam somente o apoio oferecido pelos supervisores e outros, em menor número, o apoio dos pares. A escala adaptada apresenta vantagens ao diferenciar positivas sobre a performance no trabalho de participantes de eventos instrucionais. A importância da percepção de suporte à transferência dos novos conhecimentos e habilidades incide sobre implicações práticas: o treinamento será transferido para o ambiente de trabalho à medida que essas condições tenham sido atendidas, principalmente quando satisfeitos os aspectos concernentes ao ambiente físico de trabalho e os recursos materiais imprescindíveis ao desempenho das funções, ambos de responsabilidade da organização.

Recomenda-se a aplicação da escala em organizações de trabalho, como parte integrante do instrumental na avaliação de programas de treinamento, com vistas a obter informações sobre os resultados dessas ações educacionais e os seus efeitos sobre o impacto do treinamento no trabalho. Apesar das contribuições teórico-práticas do presente estudo e da amostra com tamanho expressivo, o uso da medida adaptada pela primeira vez em uma única organização, referente a um treinamento na modalidade EAD de baixa complexidade e curta duração, impede a generalização dos resultados para outros contextos. Portanto, avaliações realizadas em contextos e amostras diferentes devem seguir atestando a capacidade preditiva da escala, podendo obter resultados diferentes, fato que acrescerá subsídios à área de avaliação de programas instrucionais. É aconselhável também o uso de mais de uma fonte de informação para contrastar as opiniões dos atores envolvidos - inclusive incluir os clientes como parte do suporte social em prestações de serviço, como é o caso do setor bancário - quanto aos 
aspectos avaliados e constatar os apoios suficientes ou deficitários influentes sobre os indicadores de efetividade de treinamento e, consequentemente, na aplicação eficaz das novas competências no trabalho.

Agradecimentos: Os autores agradecem o apoio da Coordenação de Aperfeiçoamento de Pessoal de Nível Superior (CAPES); do Grupo Tordesillas e Santander Universidades; da Fundación Carolina; do II Plan Propio de Docencia de la Universidad de Sevilla; e do Spanish Ministry of Economy and Competitiveness (MINECO, FEDER; ), número de referência do auxílio: PSI201564894-P).

\section{Referências}

Abbad, G., Pilati, R., \& Pantoja, M.J. (2003). Avaliação de treinamento: Análise da literatura e agenda de pesquisa. Revista de Administração da USP, 38(3), 205-218.

Abbad, G., \& Sallorenzo, L.H. (2001). Desenvolvimento e validação de escalas de suporte à transferência de treinamento. Revista de Administração da USP, 36(2), 33-45.

Abbad, G., Sallorenzo, L.H., Coelho, F.A., Zerbini, T., Vasconcelos, L., \& Todeschini, K. (2012). Suporte à transferência de treinamento e suporte à aprendizagem. In G. Abbad, L. Mourão, P. P. M. Meneses, T. Zerbini, J. E. Borges-Andrade, R. Vilas-Boas (Orgs.), Medidas de avaliação em treinamento, desenvolvimento e educação (pp. 127-146). Porto Alegre: Artmed.

Abbad, G., Zerbini, T., \& Souza, D.B.L. (2010). Panorama das pesquisas em educação a distância no Brasil. Estudos de Psicologia, 15(3), 291-298.

Aguinis, H., \& Kraiger, K. (2009). Benefits of training and development for individuals and teams, organizations, and society. Annual Review of Psychology, 60(1), 451-474. doi:10.1146/annurev.psych.60.110707.163505

Al-Eisa, A.S., Furayyan, M.A., \& Alhemoud, A.M. (2009). An empirical examination of the effects of self-efficacy, supervisor support and motivation to learn on transfer intention. Management Decision, 47(8), 1221-1244. doi:10.1108/00251740910984514

Alvarez, K., Salas, E., \& Garofano, C.M. (2004). An integrated model of training evaluation and effectiveness. Human Resource Development Review, 3(4), 385-416. doi: $10.1177 / 1534484304270820$

Baldwin, T.T., \& Ford, J.K. (1988). Transfer of training: A review and directions for future research. Personnel Psychology, 41(1), 63-105. doi:10.1111/j.1744-6570.1988.tb00632.x

Blume, B.D., Ford, J.K., Baldwin, T.T., \& Huang, J.L. (2010). Transfer of training: A meta-analytic review. Journal of Management, 36(4), 1065-1105. doi:10.1177/0149206309352880

Burke, L.A., \& Hutchins, H.M. (2007). Training transfer: An integrative literature review. Human Resource Development Review, 6(3), 263-297. doi:10.1177/1534484307303035

Byrne, B.M. (2010). Structural equation modeling with AMOS: Basic concepts, applications, and programming. New York/ London: Routledge Taylor \& Francis Group.

Carvalho, R.S., \& Abbad, G. (2006). Avaliação de treinamento a distância: Reação, suporte à transferência e impactos no trabalho. Revista de Administração Contemporânea, 10(1), 95-116.
Chiaburu, D.S. (2010). The social context of training: Coworker, supervisor, or organizational support?. Industrial and Commercial Training, 42(1), 53-56. doi:10.1108/00197851011013724

Chiaburu, D.S., van Dam, K., \& Hutchins, H.M. (2010). Social support in the workplace and training transfer: A longitudinal analysis. International Journal of Selection and Assessment, 18(2), 187-200. doi:10.1111/j.1468-2389.2010.00500.x

Chiaburu, D.S., \& Tekleab, A.G. (2005). Individual and contextual influences on multiple dimensions of training effectiveness. Journal of European Industrial Training, 29(8), 604-626. doi:10.1108/03090590510627085

Dermol, V., \& Čater, T. (2013). The influence of training and training transfer factors on organizational learning and performance. Personnel Review, 42(3), 324-348.

Grossman, R., \& Salas, E. (2011). The transfer of training: What really matters. International Journal of Training and Development, 15(2), 103-120. doi:10.1111/j.14682419.2011.00373.x

Joo, Y. J., Lim, K. Y., \& Park, S. Y. (2011). Investigating the structural relationships among organizational support, learning flow, learners' satisfaction and learning transfer in corporate e-learning. British Journal of Educational Technology, 42(6), 973-984.

Meneses, P.P.M., Zerbini, T., \& Martins, L.B. (2012). Determinantes situacionais e individuais da aprendizagem em ensino a distância: Desenvolvimento de escala. Psico (PUCRS), 43(2), 208-218.

Nijman, D.J.M., Nijhof, W.J., Wognum, A.A.M., \& Veldkamp, B.P. (2006). Exploring differential effects of supervisor support on transfer of training. Journal of European Industrial Training, 30(7), 529-549. doi:10.1108/03090590610704394

Pham, N.T.P., Segers, M.S.R., \& Gijselaers, W.H. (2012). Effects of work environment on transfer of training: Empirical evidence from Master of Business Administration programs in Vietnam. International Journal of Training and Development, 17(1), 1-19. doi:10.1111/j.1468-2419.2012.00417.x

Pilati, R., \& Abbad, G. (2005). Análise fatorial confirmatória da escala de impacto no treinamento no trabalho. Psicologia: Teoria e Pesquisa, 21(1), 43-51.

Pilati, R., \& Borges-Andrade, J.E. (2012). Training effectiveness: Transfer strategies, perception of support and worker commitment as predictors. Revista de Psicología del Trabajo $y$ de las Organizaciones, 28(1), 25-35. doi:10.5093/tr2012a3

Rios, J., \& Wells, C. (2014). Validity evidence based on internal structure. Psicothema, 26(1), 108-116.

Salas, E., \& Cannon-Bowers, J.A. (2001). The science of training: A decade of progress. Annual Review of Psychology, 52(1), 471-499. doi:10.1146/annurev.psych.52.1.471

Salas, E., Tannenbaum, S.I., Kraiger, K., \& Smith-Jentsch, K.A. (2012). The science of training and development in organizations: What matters in practice. Psychological Science in the Public Interest, 13(2), 74-101. doi:10.1177/1529100612436661

Tannenbaum, S.I., \& Yukl, G. (1992). Training and development in work organizations. Annual Review of Psychology, 43(1), 399-441. doi:10.1146/annurev.ps.43.020192.002151

Tracey, J.B., \& Tews, M.J. (2005). Construct validity of a general training climate scale. Organizational Research Methods, 8(4), 353-74. doi:10.1177/1094428105280055 
Van den Bossche, P., Segers, M., \& Jansen, N. (2010). Transfer of training: The role of feedback in supportive social networks. International Journal of Training \& Development, 14(2), 8194. doi:10.1111/j.1468-2419.2010.00343.x

Velada, R., Caetano, A., Michel, J.W., Lyons, B.D., \& Kavanagh, M.J. (2007). The effects of training design, individual characteristics and work environment on transfer of training. International Journal of Training and Development, 11(4), 282-294. doi:10.1111/j.1468-2419.2007.00286.x
Yarnall, J. (1998). Line managers as career developers: Rhetoric or reality?. Personnel Review, 27(5), 378-395. doi:10.1108/00483489810230325

Recebido em 22.03.2016

Primeira decisão editorial em 16.07.2016

Versão final em 12.07.2017

Aceito em 12.07.2017 


\section{APÊNDICE}

\section{SUPORTE À TRANSFERÊNCIA DE TREINAMENTO}

Os itens do questionário indicam comportamentos de supervisores e/ou pares para apoiar o uso de novas habilidades aprendidas em treinamento no ambiente de trabalho e o suporte fornecido pela organização. Utilize a escala abaixo para avaliar: a) o apoio psicossocial oferecido pelos seus supervisores e/ou pares e b) o suporte material fornecido pela organização. Leia atentamente os itens listados e escolha o ponto da escala $(1,2,3,4$ ou 5) que representa a frequência com que supervisores, pares e organização ofereceram os apoios descritos em cada item. Para avaliar o suporte psicossocial, marque sua resposta na coluna correspondente (coluna $\mathrm{S}$ = supervisores e coluna $\mathrm{P}$ = pares) e para avaliar o suporte material, registre sua resposta na coluna à direita (Nota) aos itens que descrevem tais aspectos.

\begin{tabular}{|c|c|c|c|c|}
\hline 1 & 2 & 3 & 4 & 5 \\
\hline Nunca & Raramente & Às vezes & Frequentemente & Sempre \\
\hline \hline
\end{tabular}

\begin{tabular}{|c|c|c|}
\hline \multicolumn{3}{|l|}{ Itens } \\
\hline \multicolumn{3}{|l|}{ Suporte Psicossocial } \\
\hline 1. Encorajam a aplicação no trabalho de novas habilidades aprendidas em treinamento. & $\mathrm{S}$ & $\mathrm{P}$ \\
\hline \multicolumn{3}{|l|}{ 2. Fornecem as informações necessárias ao uso eficaz das novas habilidades aprendidas em treinamento. } \\
\hline \multicolumn{3}{|l|}{ 3. Removem as dificuldades ao uso eficaz das novas habilidades aprendidas em treinamento. } \\
\hline \multicolumn{3}{|l|}{ 4. Criam oportunidades de utilizar no trabalho as novas habilidades aprendidas em treinamento. } \\
\hline \multicolumn{3}{|l|}{ 5. Fornecem feedback quanto à aplicação no trabalho das novas habilidades adquiridas em treinamento. } \\
\hline \multicolumn{3}{|l|}{ 6. Consideram minhas sugestões, em relação ao que foi aprendido no treinamento, no ambiente de trabalho. } \\
\hline Suporte Material & \multicolumn{2}{|c|}{ Notas } \\
\hline \multicolumn{3}{|l|}{$\begin{array}{l}\text { 1. São fornecidos os recursos materiais necessários para a aplicação eficaz das novas habilidades aprendidas em } \\
\text { treinamento. }\end{array}$} \\
\hline $\begin{array}{l}\text { 2. É disponibilizado local de trabalho adequado à aplicação eficaz das novas habilidades aprendidas em treinamento } \\
\text { (por exemplo, espaço físico, iluminação, mobiliário, nível de ruído). }\end{array}$ & & \\
\hline
\end{tabular}

